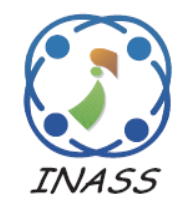

\title{
A Cost-Aware Test Suite Minimization Approach Using TAP Measure and Greedy Search Algorithm
}

\author{
Shounak Rushikesh Sugave ${ }^{1 *} \quad$ Suhas Haribhau Patil $^{2} \quad$ B. Eswara Reddy ${ }^{3}$ \\ ${ }^{I}$ MIT College of Engineering, Pune, MH, India \\ ${ }^{2}$ Bharati Vidyapeeth University College of Engineering, Pune MH, India \\ ${ }^{3}$ JNTUA College of Engineering, kalikiri, chittor district, AP, India \\ * Corresponding author's Email: shounaksugave16@gmail.com
}

\begin{abstract}
Software testing is required to detect the faults and to ensure the quality of the software under development. Usually, test suites are used to evaluate the software system during the software development cycle. But often test suites contain more redundant test cases due to overlapped test objectives. So, the test-suite reduction is an important step to reduce the number of test cases so as to satisfy the entire objectives with less computational cost. Literature presents different methods to select the suitable test suites of optimal subsets for regression testing. Accordingly, this research aims to develop an effective test suite reduction approach for regression testing. The proposed algorithm (GTAP) is newly designed using TAP measure and greedy search algorithm. This algorithm uses TAP-measure which is specially developed for measuring the importance of test cases. The performance of the GTAP algorithm is evaluated using four different evaluation metrics with eleven subject programs available in SIR repository. From the experimentation, the average performance of the proposed GTAP algorithm in all the programs is $93.07 \%$ which is higher than the DIV-GA which obtained the value of $90.27 \%$.
\end{abstract}

Keywords: Testing, Test case, Coverage, Test suite, Greedy search, Test suite reduction.

\section{Introduction}

Generally, a test suite minimization problem [15] can be simplified as a minimum set cover problem, which Karp has asserted as an NPcomplete problem. Numerous techniques have been reported in the literature. This technique exploits many software metrics to converge the test suite minimization problem to the near-optima. Test suite minimization technique produce a representative set of test cases but are unable to reduce test suite size significantly and are not efficient in reducing the execution time. W. E. Wong et al. 1995 [6] and M. J. Harrold et al. 1993 [7] have presented test suite minimization approaches for fault detection effectiveness. It is also observed that majority of the minimization algorithms did not consider the important deviations among the test cases in terms of execution penalties [1]. When these algorithms attempt to minimize the cost in terms of execution time, they fail to achieve the effective rate of fault detection. This interprets the strong need to have a technique which provides a good trade-off between the effectiveness and cost metrics such as test coverage, execution time and fault detection capability [3].

If the literature is examined, coverage can be deemed as a conventional approach that employs the Greedy Search algorithm for decreasing the number of test cases. A thorough description of the coverage specifications and the likewise test suite reduction schemes were presented in [4] and [5]. In [8], modelling-based test suite minimization problem is covered. The various search-based algorithms resulting in test suite reduction are dealt in [3, 9-18] and $[19,20]$ which briefs about several costeffective algorithms. A two-step test-suite reduction scheme is elucidated in [21] and the three weightbased Genetic Algorithms are depicted in [3]. In most of the algorithms, the test metrics allow 
choosing the test cases using decreased value of cost. Jones and Harrold [22], in their preceding work, have specified that the choice of a test case in the test suite reduction algorithms can be made in relation to its contribution or goodness. Some researchers have utilized a metric, termed as a ratio, to assess the test cases. A more recent metric, known as EIrreplaceability metric, enables decrementing the number of test cases via greedy search algorithm [1].

This paper aims to develop an effective test suite reduction approach for regression testing. Here, TAP measure is newly proposed for finding the importance of test cases using two parameters, called the number of test cases that can satisfy availed test requirement and the number of test cases that already satisfied test requirement and the GTAP algorithm is designed including TAP measure and greedy search algorithm. The proposed GTAP algorithm enables the finding of test cases using the objective measure called, TAP-measure.

In the proposed work, GTAP algorithm is devised for test suite reduction using TAP measure (Test cases which Already included in Pool-based Measure). TAP measure includes three different constraints to find the goodness of a test case. TAP measure does the selection of test cases in GTAP algorithm for multiple iterations. The selected test cases can satisfy the entire test requirement with much-reduced cost. The proposed GTAP algorithm can yield the representative set of test cases with the lowest cost and the reduction capability of the proposed algorithm is higher than the existing algorithm. The paper is organized as follows: section 2 provides the problem statement and section 3 proposes cost-aware test suite minimization approach using TAP measure and greedy search algorithm. Section 4 explains the running example and comparison and section 5 presents results and discussion. Finally, the conclusion is given in section 6 .

\section{Problem statement}

Software testing is an important field of current days due to wide usage of a software system. Before delivering any software products, testing is required to fix the faults that may exist in the module. Testing can be done through different test suite generation methods with so many techniques which are available in the literature. In [2], dubbed RZOLTAR was proposed for test suite minimization. RZOLTAR can significantly reduce the original test suite, while still maintaining the full code coverage. The drawback of this method is the test reduction which may have a negative impact on fault detection. Wang S et al. [3] have proposed a fitness function in conjunction with ten multi-objective search algorithms, to minimize the test suites in the context of product line testing. This method does not work at other industrial case studies. In [4], they introduced a family of similarity-based test case selection techniques for test suites generated from state machines called similarity-based test case selections (STCS), which minimize the similarity among selected test cases to increase the chance of detecting more faults. This method has high execution cost. Shaccour E et al. [5] presented a coverage specification language and a methodology to preserve the intent of test cases in a regression test suite. The drawback of this method is the specification language which has some weaknesses.

The minimization technique discussed in [6] can be used for more efficient program revalidation by selecting only a few effective regression tests to be re-executed after each software revision. M. J. Harrold et al. [7] presented a technique that selects a representative set of test cases from a test suite that provides the same measure of coverage as the test suite. A program modification may cause a change in a program's testing requirements. Stephen W et al. [8] proposed a static black-box TCP technique. This method does not work on distance maximization algorithms, such as hill climbing, genetic algorithms, and simulated annealing.

Here, an important consideration is how to reduce the test suite in a better way by satisfying tradeoffs: When we minimize test suites, test requirements are not satisfied completely. When we increase test suites, test requirements are satisfied but computational cost is more. To balance these two things, better methods are needed for the test suite reduction which simultaneously satisfies the test case requirement as well as cost reduction. In light constraints, few of algorithms are presented in the literature. One of the algorithms presented recently is given in [1] where, they introduced two metrics called, Irreplaceability and EIrreplaceability for test suite selection. The selection process is done with the greedy search algorithm. Their motivation of research is to improve coverage as well as cost aware-coverage for better performance it has the advantage of generating the representative set with the lowest cost because the ratio metric failed to take into consideration the above mentioned tradeoff. A representative set does yield the lowest execution cost if it includes test cases with high replaceability.

The objective of this research is to develop an effective test suite reduction approach for regression 
testing. Here, two challenges are identified, i) Right inclusion of parameters to test case metrics for test case evaluation, ii) selection of a right algorithm for searching test cases. In the previous work [1], they have additionally included a parameter called, number of test cases that satisfy in contributionTosuite. When we analyze a parameter called, EIrreplaceability [1], the degree of contribution in the overall pool is considered. But, this measure completely missed out the strategy of bringing deviation in terms of measurement among test cases which are already considered for evaluation. So, it requires a perfect mathematical formula for test suite reduction to easily select the test cases. For the second challenge, literature presents various algorithms [19-22] for reducing test suite. From the recent work [1], they proved that greedy search algorithm which seems little faster and produced good results in EIrreplaceability [1] out of other nine various algorithms. So, a greedy search algorithm is also taken here to provide a more robust solution with less computational effort.

The main contributions of the paper is given as follows,

- TAP measure is newly proposed for finding the importance of test cases using two parameters, called the number of test cases that can satisfy availed test requirement (total test cases which does not satisfy any test requirement until the current iteration) and the number of test cases that already satisfied test requirement (total test cases which already satisfy any one of test requirements until the current iteration).

- GTAP algorithm is newly designed including TAP measure and greedy search algorithm. The proposed GTAP algorithm enables the finding of test cases using the objective measure called, TAP-measure.

\section{Proposed cost-aware test suite minimization approach using TAP measure and greedy search algorithm}

This section presents the proposed method for automatic test suite selection with a novel measure for test suite evaluation using greedy search algorithm in getting the optimal solution without violating trade-offs. In this measure, a number of test requirement satisfied in previous iterations, the number of test cases that to be satisfied and cost, all are effectively included to develop new formulae for test suite evaluation. It is done with the perspective of the information-theoretic measure which can bring more deviation for every test case to easily evaluate the results. Then, a greedy search algorithm is utilized to search over the test requirement until it reaches the better trade-off.

\subsection{Test pool}

The input for the proposed test case selection is test pool which contains the test cases and its cost. A test case is a set of input variables required for software with the desired output results and test case requirement is about to employ a specific software function, loop and branch to be executed for a test case. Here, test case requirement is taken as branch coverage which provides the output whether the given test case is covered the specific branch or not. Let us assume that the number of test case for the algorithm is $n$ and number of test requirement is $m$. Then, test pool con be indicted as,

$$
T=\left\{t_{i j} \quad ; 0<i<n ; 0<j<m\right\}
$$

The cost value of each test case is computed by finding the execution time of the test case. For each test case $t$, there is an execution time which denotes the cost value of the test cases.

\subsection{New TAP measure for test suite reduction}

Test case selection is an important step in regression testing to satisfy two constraints, such as i) satisfying all test requirements, ii) Minimizing the cost value. These two constraints are very important when developing test metrics for test case selection. In this new TAP measure, the cost is inversely proportional to ratio and coverage is directly proportional to ratio.

\section{a) Existing EIrreplaceability metrics}

Recently, EIrreplaceability [1] was developed for test case selection using a parameter called, a contribution which considers the number of test cases that satisfies $r_{s}$ in the test pool. The number of test cases that satisfies the test requirement is inversely proportional to the contribution which obtains the higher value if less number of test cases can do the same task covering the test requirements. On the other hand, the high value of contribution denotes that the finding of test cases to satisfy those test requirements is tough. Along with this, the execution cost of a test is inversely included with the contribution to evaluate the test cases. Also, they assigned high priority if a test case that can only fulfill a test requirement by giving infinity. The EIrreplaceability is computed as follows: 


$$
\begin{aligned}
& \text { Contribution }(t, r s)= \begin{cases}0 & ; \text { if t cannot satisfy } r s \\
\frac{1}{\text { no. of test cases that }} & \text {; if t satisfies } r s\end{cases} \\
& \text { EIrreplaceability }(t)=\left\{\begin{array}{l}
\infty . \quad ; \text { rs can be satisfied by t only } \\
\frac{\sum_{s=1}^{m} \text { Contribution }(t, r s)}{\cos t(t)} ; \text { otherwise }
\end{array}\right.
\end{aligned}
$$

\section{b) Proposed TAP measure}

In previous work [1], EIrreplaceability selects test cases using contribution or goodness which is measured based on the characteristics of the test case whether it can satisfy the test requirement. Here, we included two parameters such as $T_{a}$ and $T_{s}$ for effective measurement of test cases. Every algorithm chooses the test cases in an iterative way of changing measures. Accordingly, Number of test cases that can satisfy availed test requirement $\left(T_{a}\right)$ and Number of test cases that already satisfied test requirement $\left(T_{s}\right)$ are the two parameters included in the proposed TAP measure. $T_{a}$ is inversely proportional to the contribution and it provides less value to the contribution if many test cases can satisfy the same test requirement. $T_{s}$ is also inversely proportional to the moving contribution but it controls the value of $T_{s}$ based on a number of test cases that already satisfied the same test requirement with decrement factor, $F_{d}$. This means that even if a test requirement is already satisfied by a test case, it can contribute to additional test requirement. If so, the value of the moving contribution for a test case is slightly reduced. The moving contribution signifies zero if the test case cannot satisfy the test requirement and a factor is computed if the test case satisfies the test requirement according to the following equation.

$$
\begin{aligned}
& C(t, r s)= \begin{cases}0 & ; \quad \text { if } t \text { cannot satisfy } r_{s} \\
\left(\frac{1}{T_{a}}\right) ; & \text { if } t \text { satisfies } r_{s}\end{cases} \\
& M C(t, r s)= \begin{cases}0 \quad ; \quad \text { if t cannot satisfy } r_{s} \\
\left(\frac{1}{F_{d} * T_{s}}\right) ; & \text { if } t \text { satisfies } r_{s}\end{cases}
\end{aligned}
$$

where $M C$ is moving contribution, $T_{a}$ is a number of test cases that can satisfy availed $r_{s}, T_{s}$ is a number of test cases that already satisfied $r_{s}$ and $F_{d}$ is decrement factor. $F_{d}$ is named as decrement factor because it reduces the moving contribution which is inversely proportional to the $T_{a} . F_{d}$ is fixed threshold which will not change into every iteration. The selection and fixing of the right value for $F_{d}$ affect the performance severely. The value suggested here is selected based on trial and error method. The numerical example shown here is the justification of fixing the value to two.

The contribution is directly proportional with TAP-measure which increases when the contribution is increased. Cost is inversely proportional to TAP-measure which increases when the contribution is decreased. Also, if any test cases that can be satisfied only one test requirement, then the value is infinity. The TAP-measure is computed as follows:

$$
T A P-\text { measure }(t)=\left\{\begin{array}{l}
\infty \quad ; \quad r_{s} \text { can be satisfied by tonly } \\
\frac{\sum_{s=1}^{k} C\left(t, r_{S}\right)-\sum_{s=1}^{l} M C\left(t, r_{S}\right)}{\cos t(t)} ; \text { otherwise }
\end{array}\right.
$$

Where $k$ is the number of test requirement availed and $l$ is the number of test requirement already satisfied.

\subsection{GTAP search algorithm for test suite reduction}

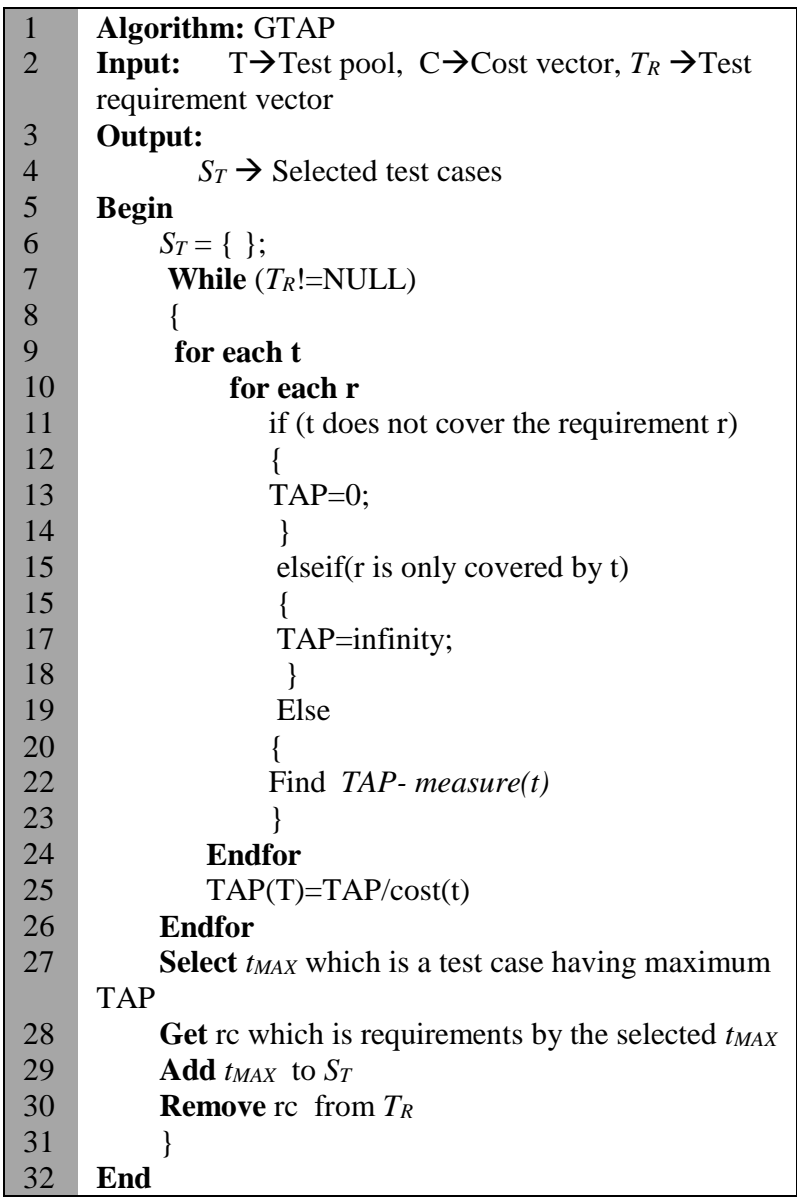

Figure. 1 GTAP search algorithm for test suite reduction

The proposed GTAP search algorithm given in Fig. 1 is used for test case selection using the 
proposed TAP measure and greedy search algorithm. The Greedy algorithm is a well-known algorithm for finding the near-optimal solution to the test suite reduction problem. This simple algorithm frequently traverses to the test pool to include uncovered test requirements from the test pool until all of the requirements are covered. The input for GTAP algorithm is a test pool $T$ which contains test cases and its coverage. The elements in the test pool have an only binary number where zero indicates that the corresponding test requirement is not solved by that test case and one indicates that the test requirement is solved by that test case. Along with the test pool, cost vector $C$ and the test requirement vector $T_{R}$ requirement are given as input to the proposed algorithm. The iterative process of the proposed algorithm is terminated only if the test requirement vector $T_{R}$ is empty.

In the initial step, TAP is found out for all the input test cases based on three constraints. Only if test case $t$ does not cover any input requirements, zero is assigned to TAP and only if test case $t$ covers only one input requirement which is not covered by any other test cases, infinity is assigned to TAP. If these two constraints are not satisfied, the usual procedure of finding $M C$ is applied and TAP measure is computed. In the second step, $t_{\text {MAX }}$ which is a test case having maximum TAP is selected from the input test pool and put it into $S_{T}$. If two test cases will have the same TAP value, we select any one of the test case. This situation will mostly happen only if the two test cases are duplicates. So, the selection of any one test case from the two test cases will not have much influence on the results. Then, a set of requirements covered by $t_{M A X}$ is given into $r c$. The solved test requirements $r c$ through test case $t_{M A X}$ is removed from the test requirement vector $T_{R}$. Then, an element which is related to satisfied test requirement is incremented with one in the input test pool. This assumption is for easily find out $T_{s}$ which is the number of test cases that already satisfied $r_{s}$ in TAP computation. This process is repeated until the termination criterion is satisfied and the selected test cases can be obtained from $S_{T}$.

\section{Running example and comparison}

This section discusses a numerical example of the greedy GreedyEIrreplaceability algorithm [5] and proposed GTAP algorithm. Table 1 shows running example of a GreedyEIrreplaceability algorithm. The input has seven test cases and seven test requirements. The cost of every test requirement is also given in the table. In the first step,
EIrreplaceability is computed for all the seven test cases. For an example, $t 1$ covers two requirements such as $r 1$ and $r 2$, where $r l$ is covered by two test cases and $r 2$ is covered by two test cases. So, the contribution is $1(1 / 2+1 / 2)$ and cost value is 1 for $t 1$. EIrreplaceability is the ratio of contribution to cost so, EIrreplaceability for the test case $t 1$ is 1 . For the test case $t 7, r 7$ is covered only by this test case so the value is assigned to infinity. Similarly, EIrreplaceability can be found out for all other test cases. After completing step 1, maximum value obtained by the test case is $t 7$ which is selected and test requirement $r 7$ which is satisfied by $t 7$ is removed from the test pool. The same procedure is continued until all the requirements are obtained. For this example, six steps are needed to obtain the entire test requirement. Finally, selected test cases are $(t 1, t 2, t 3, t 4, t 5, t 7)$ and requirements solved are $(r 1, r 2, r 3, r 4, r 5, r 6, r 7)$, The total cost required is 52 $(1+2+5+11+23+10)$ which is obtained by doing the summation of all the cost values of selected test cases.

Table 2 explains a running example of GTAP algorithm. The same input is also applied to GTAP algorithm with seven test cases and seven test requirements. The first step is exactly similar to the existing algorithm as the number of test cases that already satisfied is zero. From the first step, $r 7$ is only satisfied by the selected test case $t 7$ so the second step is also same as like the existing algorithm because we do not have availed and satisfied test requirement which contributes the value of MC to zero. Let we discuss the procedure of finding TAP measure for a test case $t 2$ as already $t 1$ and $t 7$ are selected. Test case $t 2$ can satisfy test requirements $r 2$ and $r 3$. As $r 2$ is already solved by the test case $t 1$, the number of test cases have already been satisfied by requirement $r 2$ is two and number of test cases that can satisfy availed requirement $r 3$ is two. So, $C(t, r s)$ is 0.5 and $M C(t$, $r s)$ is 0.25 when the decrement factor is fixed as two using trial and error approach. Finally, TAPmeasure for the test case $t 2$ is 0.125 which is obtained by dividing it by the cost of $t 2$. After completing step $3, t 3$ is selected but in the previous algorithm, $t 2$ is selected. The same procedure is applied until the entire test requirement is solved. The test cases selected based on this proposed procedure is $(t 1, t 3, t 5, t 7)$ which can solve all the test requirements, $R=(r 1, r 2, r 3, r 4, r 5, r 6, r 7)$. The total Cost required for this test case is only 39 $(1+5+23+10)$. 
Table 1.Running example of the GreedyEIrreplaceability algorithm

\begin{tabular}{|c|c|c|c|c|c|c|c|c|c|c|}
\hline Step 1 & & $\mathrm{r} 1$ & $\mathrm{r} 2$ & r3 & $\mathrm{r} 4$ & $\mathrm{r} 5$ & r6 & r7 & Cost & EIrreplaceability \\
\hline & $\mathrm{t} 1$ & . & . & & & & & & 1 & $\mathrm{EI}(\mathrm{t} 1)=(1 / 2+1 / 2) / 1=1$ \\
\hline & $\mathrm{t} 2$ & & . & . & & & & & 2 & $\mathrm{EI}(\mathrm{t} 2)=(1 / 2+1 / 2) / 2=0.5$ \\
\hline & $\mathrm{t} 3$ & & & . & . & & & & 5 & $\mathrm{EI}(\mathrm{t} 3)=(1 / 2+1 / 2) / 5=0.2$ \\
\hline & $\mathrm{t} 4$ & & & & . & . & & & 11 & $\mathrm{EI}(\mathrm{t} 4)=(1 / 2+1 / 2) / 11=0.09$ \\
\hline & t5 & & & & & . & . & & 23 & $\mathrm{EI}(\mathrm{t} 5)=(1 / 2+1 / 2) / 23=0.043$ \\
\hline & t6 & . & & & & & . & & 40 & $\mathrm{EI}(\mathrm{t} 6)=(1 / 2+1 / 2) / 40=0.025$ \\
\hline & t7 & & & & & & & . & 10 & $\mathrm{EI}(\mathrm{t} 7)=\infty$ \\
\hline \multirow[t]{7}{*}{ Step 2} & t1 & . & . & & & & & & & $\mathrm{EI}(\mathrm{t} 1)=(1 / 2+1 / 2) / 1=1$ \\
\hline & $\mathrm{t} 2$ & & . & . & & & & & & $E I(t 2)=(1 / 2+1 / 2) / 2=0.5$ \\
\hline & $\mathrm{t} 3$ & & & . & . & & & & & $\mathrm{EI}(\mathrm{t} 3)=(1 / 2+1 / 2) / 5=0.2$ \\
\hline & $\mathrm{t} 4$ & & & & . & . & & & & $\mathrm{EI}(\mathrm{t} 4)=(1 / 2+1 / 2) / 11=0.09$ \\
\hline & t5 & & & & & . & . & & & $\mathrm{EI}(\mathrm{t} 5)=(1 / 2+1 / 2) / 23=0.043$ \\
\hline & t6 & . & & & & & . & & & $\mathrm{EI}(\mathrm{t} 6)=(1 / 2+1 / 2) / 40=0.025$ \\
\hline & $t 7$ & & & & & & & - & & Selected \\
\hline \multirow[t]{7}{*}{ Step 3} & $t 1$ & - & - & & & & & & & Selected \\
\hline & t2 & & - & . & & & & & & $\mathrm{EI}(\mathrm{t} 2)=(1 / 2) / 2=0.25$ \\
\hline & $\mathrm{t} 3$ & & & . & . & & & & & $\mathrm{EI}(\mathrm{t} 3)=(1 / 2+1 / 2) / 5=0.2$ \\
\hline & $\mathrm{t} 4$ & & & & . & . & & & & $\mathrm{EI}(\mathrm{t} 4)=(1 / 2+1 / 2) / 11=0.09$ \\
\hline & t5 & & & & & . & . & & & $E I(t 5)=(1 / 2+1 / 2) / 23=0.043$ \\
\hline & t6 & - & & & & & . & & & $\mathrm{EI}(\mathrm{t} 6)=(1 / 2) / 40=0.012$ \\
\hline & $t 7$ & & & & & & & - & & Selected \\
\hline \multirow[t]{7}{*}{ Step 4} & $t 1$ & - & - & & & & & & & Selected \\
\hline & $t 2$ & & - & - & & & & & & Selected \\
\hline & t3 & & & - & . & & & & & $\mathrm{EI}(\mathrm{t} 3)=(1 / 2) / 5=0.1$ \\
\hline & $\mathrm{t} 4$ & & & & . & . & & & & $E I(t 4)=(1 / 2+1 / 2) / 11=0.09$ \\
\hline & t5 & & & & & . & . & & & $E I(t 5)=(1 / 2+1 / 2) / 23=0.043$ \\
\hline & t6 & - & & & & & . & & & $\mathrm{EI}(\mathrm{t} 6)=(1 / 2) / 40=0.012$ \\
\hline & $t 7$ & & & & & & & - & & Selected \\
\hline \multirow[t]{6}{*}{ Step5 } & $t 1$ & - & - & & & & & & & Selected \\
\hline & $t 2$ & & - & - & & & & & & Selected \\
\hline & t3 & & & - & - & & & & & Selected \\
\hline & t4 & & & & - & . & & & & $\mathrm{EI}(\mathrm{t} 4)=(1 / 2) / 11=0.045$ \\
\hline & $\mathrm{t} 5$ & & & & & . & . & & & $\mathrm{EI}(\mathrm{t} 5)=(1 / 2+1 / 2) / 23=0.043$ \\
\hline & t6 & - & & & & & . & & & $\mathrm{EI}(\mathrm{t} 6)=(1 / 2) / 40=0.01$ \\
\hline \multirow[t]{8}{*}{ Step6 } & $t 7$ & & & & & & & - & & Selected \\
\hline & $t 1$ & - & - & & & & & & & Selected \\
\hline & $t 2$ & & - & - & & & & & & Selected \\
\hline & $t 3$ & & & - & - & & & & & Selected \\
\hline & $t 4$ & & & & - & - & & & & Selected \\
\hline & t5 & & & & & - & . & & & $\mathrm{EI}(\mathrm{t} 5)=(1 / 2) / 23=0.021$ \\
\hline & t6 & - & & & & & . & & & $\mathrm{EI}(\mathrm{t} 6)=(1 / 2) / 40=0.0125$ \\
\hline & $t 7$ & & & & & & & - & & Selected \\
\hline
\end{tabular}

$\mathrm{S}=(\mathrm{t} 1, \mathrm{t} 2, \mathrm{t} 3, \mathrm{t} 4, \mathrm{t} 5, \mathrm{t} 7) ; \mathrm{R}=(\mathrm{r} 1, \mathrm{r} 2, \mathrm{r} 3, \mathrm{r} 4, \mathrm{r} 5, \mathrm{r} 6, \mathrm{r} 7) ;$ Total Cost $=1+2+5+11+23+10=52$

\section{Results and discussion}

This section discusses the subject programs taken for experimentation and detailed analysis of the proposed TAP measure with existing algorithm [5] using different evaluation metrics.

\subsection{Experimental setup}

\section{a) Subject programs for evaluation}

The proposed GTAP algorithm experiments with eleven subject programs taken from Softwareartifact Infrastructure Repository (SIR) [15] which contains Java, $\mathrm{C}, \mathrm{C}++$, and $\mathrm{C} \#$ programs for experimentation with testing and analysis techniques. From the repository, we have taken eleven different subject programs such as median, elevator, trityp, Apollo, pool3, printtokens, printokens2, space, replace, schedule and schedule2. The proposed algorithm is implemented in JAVA. 
Table 2.Running example of GTAP algorithm

\begin{tabular}{|c|c|c|c|c|c|c|c|c|c|c|}
\hline Step 1 & & $\mathrm{r} 1$ & $\mathrm{r} 2$ & r3 & $\mathrm{r} 4$ & r5 & r6 & r7 & Cost & TAP-measure \\
\hline & $t 1$ & . & . & & & & & & 1 & $\operatorname{TAP}(\mathrm{t} 1)=(1 / 2+1 / 2) / 1=1=1$ \\
\hline & $\mathrm{t} 2$ & & . & . & & & & & 2 & $\operatorname{TAP}(\mathrm{t} 2)=(1 / 2+1 / 2) / 2=0.5$ \\
\hline & $\mathrm{t} 3$ & & & . & . & & & & 5 & $\operatorname{TAP}(\mathrm{t} 3)=(1 / 2+1 / 2) / 5=0.2$ \\
\hline & $\mathrm{t} 4$ & & & & . & . & & & 11 & $\operatorname{TAP}(\mathrm{t} 4)=(1 / 2+1 / 2) / 11=0.09$ \\
\hline & $\mathrm{t} 5$ & & & & & . & . & & 23 & $\operatorname{TAP}(\mathrm{t} 5)=(1 / 2+1 / 2) / 23=0.043$ \\
\hline & t6 & . & & & & & . & & 40 & TAP(t6) $=(1 / 2+1 / 2) / 40=0.025$ \\
\hline & $\mathbf{t 7}$ & & & & & & & . & 10 & TAP-measure $(\mathrm{t} 7)=\infty$ \\
\hline \multirow[t]{7}{*}{ Step 2} & t1 & . & . & & & & & & & $\operatorname{TAP}(\mathrm{t} 1)=(1 / 2+1 / 2) / 1=1$ \\
\hline & $\mathrm{t} 2$ & & . & . & & & & & & $\operatorname{TAP}(\mathrm{t} 2)=(1 / 2+1 / 2) / 2=0.5$ \\
\hline & $\mathrm{t} 3$ & & & . & . & & & & & $\operatorname{TAP}(\mathrm{t} 3)=(1 / 2+1 / 2) / 5=0.2$ \\
\hline & $\mathrm{t} 4$ & & & & . & . & & & & $\operatorname{TAP}(\mathrm{t} 4)=(1 / 2+1 / 2) / 11=0.09$ \\
\hline & $\mathrm{t} 5$ & & & & & . & . & & & $\operatorname{TAP}(\mathrm{t} 5)=(1 / 2+1 / 2) / 23=0.043$ \\
\hline & t6 & . & & & & & . & & & $\operatorname{TAP}(\mathrm{t} 6)=(1 / 2+1 / 2) / 40=0.025$ \\
\hline & $t 7$ & & & & & & & - & & Selected \\
\hline \multirow[t]{7}{*}{ Step 3} & $t 1$ & - & - & & & & & & & Selected \\
\hline & $\mathrm{t} 2$ & & - & . & & & & & & $\mathrm{TAP}(\mathrm{t} 2)=0.5-(0.5 / 2) / 2=0.125$ \\
\hline & t3 & & & . & . & & & & & $\operatorname{TAP}(\mathrm{t} 3)=(1 / 2+1 / 2) / 5=0.2$ \\
\hline & $\mathrm{t} 4$ & & & & . & . & & & & $\operatorname{TAP}(\mathrm{t} 4)=(1 / 2+1 / 2) / 11=0.09$ \\
\hline & $\mathrm{t} 5$ & & & & & . & . & & & TAP(t5) $=(1 / 2+1 / 2) / 23=0.043$ \\
\hline & t6 & - & & & & & . & & & TAP $(\mathrm{t} 6)=0.5-0.25 / 40=0.00625$ \\
\hline & $t 7$ & & & & & & & . & & Selected \\
\hline \multirow[t]{7}{*}{ Step 4} & $t 1$ & - & - & & & & & & & Selected \\
\hline & $\mathrm{t} 2$ & & - & - & & & & & & $\mathrm{TAP}(\mathrm{t} 2)=0$ \\
\hline & t3 & & & - & - & & & & & Selected \\
\hline & $\mathrm{t} 4$ & & & & - & . & & & & $\mathrm{TAP}(\mathrm{t} 4)=0.5-(0.5 / 2) / 11=0.0227$ \\
\hline & t5 & & & & & . & . & & & $\mathrm{TAP}(\mathrm{t} 5)=(1 / 2+1 / 2) / 23=0.0434$ \\
\hline & t6 & - & & & & & . & & & TAP $(\mathrm{t} 6)=0.5-(0.5 / 2) / 40=0.00625$ \\
\hline & $t 7$ & & & & & & & - & & Selected \\
\hline
\end{tabular}

\section{b) Evaluation metrics}

The performance of the proposed GTAP and the existing algorithm is evaluated using the following four evaluation metrics. SuiteCost is a metric to compute the total execution time required for executing test suite. SuiteCostreduction is a metric used to compute the percentage of reduction capability of the algorithm versus the original computation time required for the input test suite. Improvement (cost) is utilized to find the cost improvement of the proposed algorithm while compared with the existing algorithm. Improvement (\%) is a percentage of improvement for the proposed algorithm in test suite reduction as compared with the existing algorithm.

$$
\operatorname{Suite} \operatorname{Cost}(T)=\sum_{t=1}^{n} \text { ExecutionTime }(t)
$$

SuiteCost $\operatorname{Re}$ duction $(S C R)=\frac{\text { SuiteCost }(T)-\text { suiteCost }(R S)}{\text { SuiteCost }(T)} \times 100 \%$

Improvemen $(\cos t)=\cos t($ algorithm 1$)-\operatorname{cost}($ algorithm 2$)$

$$
\operatorname{Improvement}(\%)=\frac{\operatorname{cost}(\text { algorithm } 1)-\operatorname{cost}(\text { algorithm } 2)}{\operatorname{cost}(\text { algorithm } 1)} \times 100 \%
$$

\subsection{Performance analysis}

The proposed GTAP algorithm is implemented using Java 1.7 with netbeans IDE 7.3. The experimentation is conducted on Windows 7 machines with Intel Core Duo processors and 2 GB of memory. The measurement of execution time and the generation of test suites are completely based on the reference paper given in [1].

\section{a) Analysis 1: Reduction capability of algorithms}

Test pool is directly given to both the algorithms, GTAP and GreedyEIrreplaceability. The ultimate aim of both the algorithms is to select test cases which should satisfy all the test requirements. Accordingly, test cases are selected by both the algorithms and the cost for all the selected test cases are computed and plotted in table 3. Reduction capability of the algorithms is analyzed through the SCR and cost. According to table 3 for $T_{e}$ of 0.5 , the proposed GTAP algorithm provides a minimum cost 
for the selected eleven programs. The total cost of $12.4 \mathrm{msec}$ for the proposed GTAP algorithm in median program as compared the value of 66.2 for the existing algorithm. Similarity, while comparing with the original cost required for all the test pool, the proposed GTAP achieved $92.7 \%$ improvement as compared with the existing algorithm which improves only $61.3 \%$. Similarly, the performance of the proposed GTAP is better than the GreedyEIrreplaceability in printtokens, printtokens 2 and space. Table 4 describe the Reduction capability of algorithms (in msec) for input $T_{e}$ of 0.75 . From table 4, the proposed GTAP obtained the cost of $5.2 \mathrm{~ms}, 3555 \mathrm{~ms}, 34 \mathrm{~ms}, 2.9 \mathrm{E} 6,1.3 \mathrm{E} 8$ against the existing algorithm which requires $28.8 \mathrm{~ms}, 53329 \mathrm{~ms}$, $74.8 \mathrm{~ms}, 5.9 \mathrm{E} 6,2.6 \mathrm{E} 8$ for the eleven subject programs. In terms of SCR, the proposed GTAP achieved the reduction improvement of 96.6\%, 93.9, $74.2 \%, 54.6$ and $98.18 \%$ against the existing algorithm which reached the improvement of $81.7 \%$, $9.4 \%, 44.2 \%, 8.1 \%$ and $96.3 \%$. Overall, the proposed algorithm proved that reduction of test suites is much possible as compared with the existing algorithm.

Table 3. Reduction capability of algorithms (in msec) for input threshold $(\mathrm{Te})$ of 0.5

\begin{tabular}{|l|l|l|l|l|l|}
\hline Program & Original & $\begin{array}{l}\text { GTAP } \\
\text {-Cost }\end{array}$ & $\begin{array}{l}\text { GreedyEIr } \\
\text { replaceabil } \\
\text { ity-Cost }\end{array}$ & $\begin{array}{l}\text { GTAP- } \\
\text { SCR }\end{array}$ & $\begin{array}{l}\text { IrreedyE } \\
\text { ability- } \\
\text { SCR }\end{array}$ \\
\hline Median & 171.3 & 12.4 & 66.2 & 92.7 & 61.3 \\
\hline Elevator & 115359.3 & 7201.3 & 107976.5 & 93.7 & 6.3 \\
\hline Trityp & 436.8 & 7.6 & 68.6 & 98.2 & 84.2 \\
\hline Apollo & $6.5 \mathrm{E} 6$ & $2.9 \mathrm{E} 6$ & $6.0 \mathrm{E} 6$ & 54.3 & 7.6 \\
\hline Pool3 & $1.7 \mathrm{E} 11$ & $1.0 \mathrm{E} 8$ & $2.0 \mathrm{E} 8$ & 99.9 & 99.8 \\
\hline printtokens & 1350 & 40.8 & 69.4 & 96.9 & 94.8 \\
\hline printtokens2 & 932 & 46.6 & 46.7 & 94.9 & 94.9 \\
\hline Space & 106281 & 118.72 & 211.6 & 99.88 & 99.8 \\
\hline replace & 2143 & 113.87 & 109.36 & 94.68 & 94.9 \\
\hline schedule & 613 & 36.18 & 38.16 & 94.0 & 93.68 \\
\hline Schedule2 & 845 & 42.08 & 43.52 & 95.02 & 94.85 \\
\hline
\end{tabular}

Table 4. Reduction capability of algorithms (in msec) for input threshold of 0.75

\begin{tabular}{|l|l|l|l|l|l|}
\hline Program & $\begin{array}{c}\text { Origin } \\
\text { al }\end{array}$ & $\begin{array}{c}\text { GTAP- } \\
\text { Cost }\end{array}$ & $\begin{array}{c}\text { GreedyEIrr } \\
\text { eplaceabilit } \\
\text { y-Cost }\end{array}$ & $\begin{array}{c}\text { GTAP } \\
\text {-SCR }\end{array}$ & $\begin{array}{c}\text { IreedyE } \\
\text { eability- } \\
\text { SCR }\end{array}$ \\
\hline Median & 157.9 & 5.2 & 28.8 & 96.6 & 81.7 \\
\hline elevator & 58896.9 & 3555.3 & 53329.4 & 93.9 & 9.4 \\
\hline trityp & 134.4 & 34.5 & 74.8 & 74.2 & 44.2 \\
\hline Apollo & $6.4 \mathrm{E} 6$ & $2.9 \mathrm{E} 6$ & $5.9 \mathrm{E} 6$ & 54.6 & 8.1 \\
\hline Pool3 & $7.3 \mathrm{E} 9$ & $1.3 \mathrm{E} 8$ & $2.6 \mathrm{E} 8$ & 98.1 & 96.3 \\
\hline printtokens & 1341 & 39.8 & 53.4 & 97 & 96.0 \\
\hline printtokens2 & 921 & 44.6 & 45.1 & 95.1 & 95.1 \\
\hline space & 105186 & 116 & 210 & 99.88 & 99.80 \\
\hline replace & 21455 & 118 & 115 & 99.45 & 99.46 \\
\hline schedule & 650 & 38 & 36 & 94.15 & 94.46 \\
\hline Schedule2 & 827 & 50 & 44 & 93.95 & 94.67 \\
\hline
\end{tabular}

Table 5. Relative Reduction capability of algorithms for input threshold 0.5

\begin{tabular}{|l|l|l|l|l|}
\hline Program & $\begin{array}{l}\text { GTAP- } \\
\text { Cost }\end{array}$ & $\begin{array}{l}\text { GreedyEIrrepl } \\
\text { aceability-Cost }\end{array}$ & $\begin{array}{l}\text { Improvem } \\
\text { ent(cost) }\end{array}$ & $\begin{array}{l}\text { Improvem } \\
\text { ent (\%) }\end{array}$ \\
\hline Median & 12.4 & 66.2 & 53.7 & 81.1 \\
\hline elevator & 7201.3 & 107976.5 & 100775.2 & 93.3 \\
\hline trityp & 7.6 & 68.6 & 60.9 & 88.8 \\
\hline Apollo & $2.9 \mathrm{E} 6$ & $6.0 \mathrm{E} 6$ & $3.0 \mathrm{E} 6$ & 50.5 \\
\hline Pool3 & $1.0 \mathrm{E} 8$ & $2.0 \mathrm{E} 8$ & $1.0 \mathrm{E} 8$ & 50.2 \\
\hline printtokens & 40.8 & 69.4 & 28.6 & 41.1 \\
\hline printtokens2 & 46.1 & 46.7 & 0.1 & 0.2 \\
\hline space & 118.72 & 211.6 & 92.8 & 43.8 \\
\hline replace & 113.87 & 109.36 & 4.51 & 3.96 \\
\hline schedule & 36.18 & 38.16 & 1.98 & 3.13 \\
\hline Schedule2 & 42.08 & 43.52 & 1.44 & 3.42 \\
\hline
\end{tabular}

Table 6. Relative Reduction capability of algorithms for input threshold 0.75

\begin{tabular}{|l|l|l|l|l|}
\hline Program & $\begin{array}{c}\text { GTAP- } \\
\text { Cost }\end{array}$ & $\begin{array}{l}\text { GreedyEIrrepl } \\
\text { aceability-Cost }\end{array}$ & $\begin{array}{c}\text { Improvem } \\
\text { ent (cost) }\end{array}$ & $\begin{array}{l}\text { Improvem } \\
\text { ent }(\%)\end{array}$ \\
\hline Median & 5.2 & 28.8 & 23.5 & 81.6 \\
\hline elevator & 3555.3 & 53329.4 & 49774.186 & 93.3 \\
\hline trityp & 34.5 & 74.8 & 40.32 & 53.8 \\
\hline Apollo & $2.9 \mathrm{E} 6$ & $5.9 \mathrm{E} 6$ & $3.0 \mathrm{E} 6$ & 50.5 \\
\hline Pool3 & $1.3 \mathrm{E} 8$ & $2.6 \mathrm{E} 8$ & $1.3 \mathrm{E} 8$ & 50.2 \\
\hline printtokens & 39.8 & 53.4 & 13.5 & 25.4 \\
\hline printtokens2 & 44.6 & 45.1 & 0.5 & 1.1 \\
\hline space & 116 & 210 & 94 & 44.7 \\
\hline replace & 118 & 115 & 3 & 2.5 \\
\hline schedule & 38 & 36 & 2 & 5.26 \\
\hline Schedule2 & 50 & 44 & 6 & 12 \\
\hline
\end{tabular}

Table 7. Reduced test suite size of algorithms for input threshold 0.5

\begin{tabular}{|l|l|l|l|}
\hline Program & $\begin{array}{l}\text { Original } \\
\text { test suite }\end{array}$ & $\begin{array}{l}\text { GTAP- } \\
\text { suite size }\end{array}$ & $\begin{array}{l}\text { GreedyEIrre } \\
\text { placeability- } \\
\text { suite size }\end{array}$ \\
\hline Median & 150 & 10 & 58 \\
\hline elevator & 2500 & 156 & 2340 \\
\hline Trityp & 300 & 5 & 47 \\
\hline Apollo & 20000 & 9130 & 18500 \\
\hline Pool3 & 10000 & 5 & 11 \\
\hline printtokens & 1200 & 40 & 64 \\
\hline printtokens2 & 1100 & 56 & 56 \\
\hline Space & 6300 & 100 & 200 \\
\hline replace & 5211 & 425 & 430 \\
\hline schedule & 2254 & 305 & 310 \\
\hline schedule2 & 2345 & 240 & 250 \\
\hline
\end{tabular}

Table 8. Reduced test suite size algorithms for input threshold 0.75

\begin{tabular}{|l|l|l|l|}
\hline Program & $\begin{array}{c}\text { Original } \\
\text { test suite }\end{array}$ & $\begin{array}{c}\text { GTAP- } \\
\text { suite size }\end{array}$ & $\begin{array}{c}\text { GreedyEIrre } \\
\text { placeability- } \\
\text { suite size }\end{array}$ \\
\hline Median & 145 & 4 & 26 \\
\hline Elevator & 2300 & 139 & 2082 \\
\hline Trityp & 2850 & 73 & 158 \\
\hline Apollo & 19000 & 8620 & 17444 \\
\hline Pool3 & 9160 & 166 & 333 \\
\hline Printtokens & 1110 & 40 & 64 \\
\hline printtokens2 & 1021 & 55 & 56 \\
\hline Space & 5100 & 97 & 197 \\
\hline replace & 5301 & 601 & 625 \\
\hline schedule & 2120 & 254 & 263 \\
\hline schedule2 & 2125 & 220 & 231 \\
\hline
\end{tabular}


Table 9. Comparative discussion with existing algorithms

\begin{tabular}{|l|l|l|l|l|}
\hline Program & $\begin{array}{l}\text { GreedyCo } \\
\text { verage [1] }\end{array}$ & $\begin{array}{l}\text { GreedyEIrrepl } \\
\text { aceability [1] }\end{array}$ & $\begin{array}{l}\text { DIVGA } \\
{[26]}\end{array}$ & GTAP \\
\hline Median & 80.2 & 81.7 & 80.5 & $\mathbf{9 6 . 6}$ \\
\hline Elevator & 8.3 & 9.4 & 85.36 & $\mathbf{9 3 . 9}$ \\
\hline Trityp & 83.5 & 84.2 & 83.75 & $\mathbf{9 8 . 2}$ \\
\hline Apollo & 80.2 & 8.1 & 80.3 & $\mathbf{5 4 . 6}$ \\
\hline Pool3 & 85.3 & 99.8 & 87.8 & $\mathbf{9 9 . 9}$ \\
\hline Printtokens & 96.76 & 96.0 & 96.98 & $\mathbf{9 7}$ \\
\hline printtokens2 & 94.83 & 95.1 & 94.88 & $\mathbf{9 5 . 1}$ \\
\hline Space & 99 & 99.80 & 99.8 & $\mathbf{9 9 . 8 8}$ \\
\hline replace & 94.9 & 99.46 & 94.68 & 99.45 \\
\hline schedule & 93.68 & 94.46 & 94 & 94.15 \\
\hline schedule2 & 94.85 & 94.85 & 95.02 & $\mathbf{9 5 . 0 2}$ \\
\hline
\end{tabular}

\section{b) Analysis 2: Relative Reduction capability of algorithms}

Table 5 provides Relative Reduction capability of algorithms for input threshold of 0.5 . Relative Reduction capability provides the improvement of the proposed algorithm with respect to the existing algorithm. From table 5, the proposed GTAP achieved the cost improvement of 53.7, 1007755.2, $60.9,3041487.1$ and 1.0E8 for all the subject programs. The percentage of improvement for the proposed GTAP as compared with existing one is $81.1 \%, 93.3 \%, 88.8 \%, 50.5 \%$ and $50.2 \%$ in all the programs. When analyzing the proposed GTAP with respect to the GreedyEIrreplaceability in printtokens, the proposed GTAP shows the cost value of 40.8 as compared with the value of 69.4 which is obtained by GreedyEIrreplaceability. In terms of improvement $(\%)$, the proposed shows the better value of $41.1 \%$ and $0.2 \%$ for printtokens and printtokens2. Overall, the proposed GTAP shows the better performance than the existing method. Table 6 shows the Relative Reduction capability of algorithms for input threshold of 0.75 . The improvement is $81.6 \%, 93.3 \%, 53.8 \%, 50.5 \%$ and $50.2 \%$ for median, elevator, trityp, Apollo and pool3 programs. For input threshold of 0.75 , the cost improvement is 23.5, 49774, 40.3, 3E6 and 1.3E8 for Median, elevator, trityp, Apollo, pool3 programs.

\section{c) Analysis 3: Reduced size of test suite}

From the table 7, we understand that the original test suite is reduced from 150 to 10 for median program if the proposed GTAP algorithm is applied. On the other hand, the suite size is reduced from 20,000 to 9130 for the Apollo program. For printtokens, the original test suite size of 1200 is reduced to 40 for the proposed GTAP and 64 for the GreedyEIrreplaceability. This analysis clearly shows the size of the test suite is comparatively less for the proposed GTAP algorithm as compared with GreedyEIrreplaceability. Similarly, Table 8 shows the reduced test suite size of algorithms for input threshold 0.75 . This table clearly indicates that the original test suite of 9160 is reduced to 166 for GTAP and 333 for the GreedyEIrreplaceability in Pool3. Also, for printtokens2, the test suite size of 1021 is reduced to 55 for the proposed GTAP and 56 for GreedyEIrreplaceability.

\section{d) Comparative discussion}

Table 9 provides the comparative discussion of the proposed algorithm with the existing algorithms such as, GreedyCoverage [1], GreedyEIrreplaceability [1] and DIVGA [26]. In terms of SCR, the proposed GTAP achieved the reduction improvement of $96.6 \%, 93.9 \%, 98.2 \%$, $54.6 \%, 99.9 \%, 97 \%, 95.1 \%, 99.88 \%, 99.45 \%$, $94.15 \%$, and $95.02 \%$ for all the programs Median, Elevator, Trityp, Apollo, Printtokens, Printtokens2, Space, replace, schedule, and schedule 2 respectively. The GreedyCoverage algorithm achieves the reduction improvement of $80.2 \%, 8.3 \%, 83.5 \%$, $80.2 \%, 85.3 \%, 96.76 \%, 94.83 \%, 99 \%, 94.9 \%$, $93.68 \%, 94.85 \%$ for all the subject programs. The GreedyEIrreplaceability algorithm achieves the reduction improvement of $81.7 \%, 9.4 \%, 84.2 \%$, $8.1 \%, 99.8 \%, 96.0 \%, 95.1 \%, 99.80 \%, 99.46 \%$, $94.46 \%, 94.85 \%$ for all the programs. Overall, the proposed GTAP algorithm proved that the test suite is much reduced as compared with the existing algorithms. The reason of this much reduction is happened because of the TAP measure integrated within the greedy algorithm.

\section{Conclusion}

We have presented an effective test case reduction approach for regression testing using TAP measure and greedy search algorithm. The proposed test reduction approach, called GTAP algorithm is newly designed including two additional parameters such as, the number of test cases that can satisfy much availed test requirement and number of test cases that already satisfied test requirement for identifying the important test cases available in test suite. Also, the proposed GTAP algorithm can yield the representative set of test cases with the lowest cost. The experimentation of the proposed algorithm is done using eleven subject programs available in SIR repository. The effectiveness of the proposed GTAP and the existing algorithm is evaluated using reduction capability and relative capability. From the experimentation, the average performance of the proposed GTAP algorithm in all the programs is 93.07\% which is higher than the DIV-GA which obtained the value of $90.27 \%$. The future work can be done using an optimization algorithm to select 
test cases more optimally and the fault detection capability will be extensively studied based on the reduced test suite.

\section{References}

[1] C.Lin, K.Tang, and G.M. Kapfhammer, "Test suite reduction methods that decrease regression testing costs by identifying irreplaceable tests", Information and Software Technology, Vol. 56, pp. 1322-1344, 2014.

[2] J.Campos and R.Abreu, "Encoding Test Requirements as Constraints for Test Suite Minimization", in proceedings of 10th International Conference on Information Technology: New Generations, 2013.

[3] S.Wang, S.Ali, and A.Gotlieb, "Cost-effective test suite minimization in product lines using search techniques", The Journal of Systems and Software, pp. 1-22, 2014.

[4] H.Hemmati, A. Arcuri, and L.Briand, "Achieving Scalable Model-Based Testing Through Test Case Diversity", ACM Transactions on Software Engineering and Methodology (TOSEM), Vol. 22, No. 1, 2013.

[5] E. Shaccour, F.Zaraket, and W.Masri, "Coverage Specification for Test Case Intent Preservation in Regression Suites", in proceedings of IEEE Sixth International Conference on Software Testing, Verification and Validation Workshops, 2013.

[6] W. E. Wong, J .R. Horgan, S. London, and A. P. Mathur, "Effect of Test Set Minimization on Fault Detection Effectiveness," in Proceedings of the 17th International Conference on Software Engineering (ICSE), Seattle, WA, USA, pp. 41-50, April 1995.

[7] M. J. Harrold, R. Gupta, and M. L. Soffa, "A Methodology for Controlling the Size of A Test Suite," ACM Transactions on Software Engineering and Methodology, Vol. 2, No.3, pp. 270-285, July 1993.

[8] W.Stephen, T.Hemmati, A.E. Hassan and D. Blostein, "Static test case prioritization using topic models", Empir Software Eng, Vol. 19, pp. 182-212, 2014.

[9] M.Harman, S.A. Mansouri and Y.Zhang, "Search Based Software Engineering: Trends, Techniques and Applications", Journal ACM Computing Surveys(CSUR), Vol.45, No.1, 2012.

[10] R.E. Opez-Herrejon, F.Chicano, J.Ferrer, A.Egyed and E.Alba, "Multi-objective optimal test suite computation for software product line pairwise testing", International Conference on
Software Maintenance (ICSM), pp. 404-407, 2013.

[11] S.Yoo, and M.Harman, "Using hybrid algorithm for Pareto efficient multi-objective test suite minimisation", J. Syst. Softw., Vol. 83, No. 4, pp. 689-701, 2010.

[12] A.Arcuri and L.Briand, "A practical guide for using statistical tests to assess randomized algorithms in software engineering", $33^{\text {rd }}$ ACM/IEEE International Conference on Software Engineering, ACM, pp. 1-10, 2011.

[13] Software-artifact Infrastructure Repository (SIR), http://sir.unl.edu/content/sir.php.

[14] T.Wang, R.Gao, Z.Chen, E. Wong and B. Luo, "WAS: A weighted attribute-based strategy for cluster test selection", Journal of Systems and Software, Vol. 98, pp. 44-58, 2014.

[15] G.Fraser, A.Arcuri and P. McMinn, "A Memetic Algorithm for whole test suite generation", Journal of Systems and Software, Vol. 103, pp. 311-327, 2015.

[16] S.Sampath and R.C. Bryce, "Improving the effectiveness of test suite reduction for usersession-based testing of web applications", Information and Software Technology, Vol. 54, No. 7, pp. 724-738, 2012.

[17] J.Lin and C.Huang, "Analysis of test suite reduction with enhanced tie-breaking techniques", Information and Software Technology, Vol. 51, No. 4, pp. 679-690, 2009.

[18] I. Rodriguez, L.Llana and P.Rabanal, , "A General Testability Theory: Classes, Properties, Complexity, and Testing Reductions", IEEE Transactions on Software Engineering, Vol. 40, No. 9, pp. 862 - 894, 2014.

[19] D.Jeffrey and N.Gupta, "Improving fault detection capability by selectively retaining test cases during test suite reduction", IEEE Trans. Softw. Eng., Vol. 33, No. 2, pp. 108-123, 2007.

[20] C.T.Lin, K.W.Tang, C.D.Chen and G.M. Kapfhammer, "Reducing the cost of regression testing by identifying irreplaceable test cases", 6th International Conference on Genetic and Evolutionary Computing.

[21] G.Dandan, W.Tiantian, S.Xiaohong, M.Peijun, "A test-suite reduction approach to improving fault-localization effectiveness", Computer Languages, Systems \& Structures, Vol. 39, pp. 95-108, 2013.

[22] J.A.Jones and M.J.Harrold, "Test-suite reduction and prioritization for modified condition/decision coverage", IEEE Transaction on Software Engineering, Vol. 29, No. 3, pp. 195-200, 2003. 